Nota técnica

Volumen 32(3):991-999. Septiembre-diciembre, 2021 e-ISSN 2215-3608, doi:10.15517/am.v32i3.41394

https://revistas.ucr.ac.cr/index.php/agromeso/index

\title{
Actividad herbicida de tres productos naturales sobre cuatro especies de arvenses $^{1}$
}

\section{Herbicidal activity of three natural products on four weed species}

\author{
Mary Pamela Portuguez-García², Renán Agüero-Alvarado², María Isabel González-Lutz ${ }^{3}$
}

1 Recepción: 12 de agosto, 2020. Aceptación: 3 de marzo, 2021. Este trabajo formó parte del proyecto presentado en la Vicerrectoría de Acción Social de la Universidad de Costa Rica ED-1816 nombrado: Clínica de diagnóstico, capacitación y consultoría en plantas arvenses.

2 Universidad de Costa Rica, Estación Experimental Fabio Baudrit Moreno, Programa de Malezas. Alajuela, Costa Rica.mary.portuguez@ucr.ac.cr (autora para la correspondencia; https://orcid.org/0000-0002-3520-7699), raguerofbm@ gmail.com (https://orcid.org/0000-0001-9053-9998).

3 Universidad de Costa Rica, Escuela de Estadística. San José, Costa Rica. mariaisabel.gonzalezlutz@ucr.ac.cr (https://orcid.org/0000-00023073-7746).

\section{Resumen}

Introducción. Ciertas sustancias de origen natural pueden tener actividad herbicida sobre algunas especies de arvenses. Objetivo. Evaluar la eficacia de tres sustancias de origen natural para el control de cuatro especies de arvenses y compararla con el efecto de un herbicida sintético de amplio espectro. Materiales y métodos. En febrero del 2017 se condujo un experimento en un invernadero en la Estación Experimental Agrícola Fabio Baudrit Moreno, de la Universidad de Costa Rica. Se evaluaron tres productos comerciales de origen natural a base de: d-limoneno al $55 \%$, extracto de pino al $15 \%$ (Pinus sp.) y extracto de tomillo al $23 \%$ (Thymus vulgaris); además, se evaluó un producto de origen sintético, glufosinato de amonio 14 SL. Se incluyó un testigo absoluto. Estos se asperjaron sobre plantas de Bidens pilosa, Amaranthus sp., Echinochloa colona y Rottboellia cochinchinensis. Resultados. Las plantas asperjadas con los herbicidas naturales mostraron síntomas a las dos horas, pero su eficacia final varió entre especies. A los ocho días después de la aspersión el d-limoneno resultó más eficaz que todos los demás tratamientos, mostró grados de daño mayores a 4,25 en todas las especies. Con el extracto de pino y el extracto de tomillo no se obtuvo eficacia sobre B. pilosa. El extracto de pino causó mayores daños en Amaranthus sp, E. colona y R. cochinchinensis, mientras que el extracto de tomillo en Amaranthus sp y $R$. cochinchinensis. El herbicida sintético glufosinato mostró daños significativos en las cuatro especies. Conclusiones. Los productos comerciales de origen natural utilizados, tuvieron actividad herbicida sobre las cuatro especies de arvenses evaluadas.

Palabras clave: herbicidas naturales, glufosinato, d-limoneno, extracto de pino, extracto de tomillo.

\begin{abstract}
Introduction. Certain naturally derived substances may have herbicidal activity on some weeds species. Objective. To evaluate the efficacy of three substances of natural origin for the control of four weed species and to compare it with the effect of a synthetic broad-spectrum herbicide. Materials and methods. In February 2017, an experiment was conducted in a greenhouse at the Fabio Baudrit Moreno Agricultural Experiment Station, Universidad de Costa Rica. Three commercial products of natural origin based on: $55 \%$ d-limonene, $15 \%$ pine extract (Pinus sp.)
\end{abstract}


and 23\% thyme extract (Thymus vulgaris) were evaluated; additionally, a product of synthetic origin, gluphosinate ammonium 14 SL, was evaluated. A none treated check was included. These were sprayed on seedlings of Bidens pilosa, Amaranthus sp., Echinochloa colona and Rottboellia cochinchinensis. Results. The seedlings sprayed with the natural herbicides showed symptoms at two hours, but their final efficacy varied among species. Eight-days after spraying, d-limonene was more effective than all other treatments, showing damage ratings higher than 4.25 in all species. Pine and thyme extracts were not effective on $B$. pilosa. Pine extract caused greater damage on Amaranthus $\mathrm{sp}$, E. colona, and $R$. cochinchinensis, while the thyme extract caused greater damage on Amaranthus $\mathrm{sp}$ and $R$. cochinchinensis. The synthetic herbicide gluphosinate showed significant damage on all four species. Conclusions. The commercial products of natural origin used had herbicidal activity on the four weed species tested.

Keywords: natural herbicides, glufosinate, d-limonene, pine extract, thyme extract.

\section{Introducción}

El uso de sustancias naturales con perfil ambiental y toxicológico más seguro es una necesidad creciente, pues las personas consideran que los productos naturales son más seguros que los herbicidas convencionales, aunque esto aún no se ha validado. Es una de las razones para examinar, investigar y luego usar estos productos en la agricultura convencional y orgánica (Dayan \& Duke, 2014).

La literatura reporta herbicidas naturales que poseen nuevos modos de acción, no obstante, no se utilizan de forma comercial (Dayan \& Duke, 2014). Esto se debe a varias razones: 1) en general, las moléculas bioherbicidas poseen una estructura molecular más compleja en comparación con los herbicidas sintéticos, por ello, se requieren diferentes enfoques para generar herbicidas comerciales de este tipo (Duke et al., 2000), 2) no se pueden obtener los compuestos en suficiente cantidad, 3) algunas sustancias resultan tóxicas o muy volátiles, lo cual impide su estudio (Duke \& Lydon, 1987), 4) hay un costo significativo asociado al desarrollo de un bioherbicida (Bailey, 2014) y 5) los herbicidas naturales disponibles tienen poca o ninguna selectividad y deben asperjarse en dosis muy altas para que sean eficaces (Dayan et al., 2009).

Existe una amplia gama de herbicidas sintéticos cuya aplicación, a pesar de su eficacia, trae ciertas desventajas como contaminación de los suelos y desequilibrio ambiental (Bhowmik \& Inderjit, 2003; Blanco, 2006). Su uso frecuente y la aplicación de altas dosis han desencadenado la resistencia en arvenses, que reduce la eficacia del control (Valverde \& Heap, 2009). Por esta razón, el uso de herbicidas naturales podrían ser una alternativa para el control de arvenses, ya que, en general, se reportan como biodegradables y ofrecen una mayor cantidad de modos de acción específicos, en comparación con los herbicidas de origen sintético (Bhowmik \& Inderjit, 2003; Dayan \& Duke, 2014). Con el uso de diversas prácticas de manejo se puede consolidar un manejo de arvenses sostenible y eficaz (Jabran et al., 2015). Entre las prácticas alternativas, se encuentra el uso de extractos de plantas que desprenden sustancias alelopáticas (Bhowmik \& Inderjit, 2003).

El d-limoneno es una sustancia que se extrae de la cáscara de naranja y se usa como un solvente, forma parte de grupo de hidrocarburos no saturados y es sensible a procesos de oxidación y polimerización que originan productos oscuros e insolubles (Masschelein, 2004). Se emplea como solvente, agente de limpieza, aditivo, saborizante en productos alimenticios, aromatizante, pesticida y herbicida natural. En este último caso se usa como un herbicida de contacto (Dotolo, 1983; Messerschmidt \& Jankauskas, 2012; Shrestha et al., 2012). El limoneno en concentraciones de 0,1 y $0,5 \mu \mathrm{l}$ afectó la germinación y a $0,7 \mu \mathrm{l}$ y el crecimiento de la arvense Amaranthus viridis L, también se reportó una disminución de su clorofila y respiración (Vaid et al., 2011). 
El extracto de Pinus caribaea Morelet en dosis al $100 \%$ (1 kg de acículas de pino por cada 101 de agua), inhibió en un $53 \%$ la germinaciónde arvenses asociadas a un ambiente controlado; asimismo, esa misma especie de pino redujo la brotación de arvenses presentes en café bajo sombra (Jiménez-Ferrer et al., 2006; Hernández \& Álvarez, 2008).

El aceite esencial de tomillo (Thymus vulgaris), produjo efecto fitotóxico con dosis del 1 al $2 \%(\mathrm{v} / \mathrm{v})$ al causar alteraciones en la permeabilidad de la membrana de las especies de arvenses: Taraxacum officinale L., Chenopodium album L., Ambrosia artemisiifolia L. y Sorghum halepense L. (Tworkoski, 2002).

El objetivo del trabajo fue evaluar la eficacia de tres sustancias de origen natural para el control de cuatro especies de arvenses y compararla con el efecto de un herbicida sintético de amplio espectro.

\section{Materiales y métodos}

El experimento se llevó a cabo en condiciones controladas en una casa malla de la Estación Experimental Agrícola Fabio Baudrit Moreno (EEAFBM), ubicada en La Garita de Alajuela, Costa Rica, entre las coordenadas latitud $\mathrm{N} 10^{\circ} 00373^{\prime}$ y longitud $\mathrm{W} 084^{\circ} 15.992^{\prime}$, a una temperatura promedio de $22^{\circ} \mathrm{C}$ y una altitud de $840 \mathrm{~m}$.

En el mes de febrero del 2017 se seleccionaron cuatro especies de arvenses, dos de hoja ancha (Bidens pilosa y Amaranthus sp.) y dos de hoja angosta (Echinochloa colona y Rottboellia cochinchinensis). Las semillas de $B$. pilosa, Amaranthus sp. y R. cochinchinensis, se recolectaron de la EEAFBM en febrero del 2013, noviembre del 2015 y abril del 2013, respectivamente; las de E. colona se recolectaron de un lote ubicado en Filadelfia, Carrillo, Guanacaste en septiembre del 2016.

Las semillas de cada población se embebieron en una solución de nitrato de potasio $\left(\mathrm{KNO}_{3}\right)$ al $0,25 \%$ durante 24 horas y luego de su germinación se realizó un almácigo. Se trasplantaron seis plántulas de cada especie en un macetero de $17 \mathrm{~cm}$ de diámetro, con suelo esterilizado. Según el procedimiento de Castillo \& Gómez (2016). Las plantas se mantuvieron a capacidad de campo con riegos programados por goteo.

La aspersión de los herbicidas se realizó en posemergencia temprana a los ocho días después del trasplante de las arvenses, cuando las especies tenían de dos a tres hojas verdaderas. Se emplearon cuatro herbicidas, tres de origen natural (productos comerciales), extracto de pino (Pinus sp.) al $15 \%$ (5,25 kg ia ha $\left.{ }^{-1}\right)$ (Skat orgánico, s.f.), extracto de tomillo (Thymus vulgaris) al $23 \%\left(2,41 \mathrm{~kg}^{\text {ia ha }}{ }^{-1}\right.$ ) (Agro Research International, 2020), d-limoneno al $55 \%\left(26,95 \mathrm{~kg}\right.$ ia ha-1) (Avenger, s.f.) y uno de origen sintético, glufosinato de amonio $14 \mathrm{SL}\left(0,39 \mathrm{~kg} \mathrm{ia} \mathrm{ha}^{-1}\right)$ (Bayer AG, 2020). Se incluyó, además, un testigo sin aplicación de herbicida y sin adherente. A cada caldo de aplicación se le adicionó un adherente (alcohol isotridecílico etoxilado) al 0,09\% (\%v/v) (Enlasa, s.f.). Para la aspersión se utilizó una bomba de espalda manual de 18 1, afuera de la casa de malla. La descarga promedio de la boquilla fue de $800 \mathrm{ml} \mathrm{min}^{-1}$ y la velocidad de aspersión fue de $1 \mathrm{~m} \mathrm{~s}^{-1}$. El volumen de aspersión fue de $2501 \mathrm{ha}^{-1}$. A los ocho y quince días después de la aplicación (dda), se evaluaron dos variables de respuesta, número de plantas muertas y grado de daño ( $1=$ sin síntomas aparentes, $2=$ presencia de síntomas leves (clorosis leve), 3= síntomas moderados (clorosis generalizada), $4=$ síntomas severos (clorosis generalizada, con algo de tejido necrótico) y $5=$ síntomas muy severos (similar a 4, pero con frecuente muerte de plantas) (modificado de European Weed Research Society) (Sandral et al., 1997).

El diseño del experimento fue un modelo irrestricto al azar con un arreglo factorial de dos factores: tipo de herbicida y tipo de arvense, a cuatro niveles cada uno. Los tratamientos se replicaron cuatro veces (en total 80 unidades experimentales). La variable de respuesta grado de daño, se analizó con análisis de varianza y contrastes ortogonales. El número de plantas muertas se analizó mediante un modelo de regresión logística. Se utilizó el programa estadístico JMP versión 9. 


\section{Resultados}

\section{Grado de daño}

Para el grado de daño la interacción herbicida*especie resultó significativa en ambas fechas, por lo que el análisis se hizo al comparar el efecto de los herbicidas para cada especie o la diferencia del efecto de un herbicida dado de una especie a otra. Los dos enfoques proporcionaron información interesante, por lo que se decidió estudiar ambos (Cuadro 1 y 2).

Cuadro 1. Grado de daño promedio a los ocho días después de aspersión de sustancias de origen natural para el control de cuatro especies de arvenses, por especie, por herbicida y las probabilidades asociadas a los contrastes de hipótesis entre especies para cada herbicida y entre herbicidas para cada especie. EEAFBM, Alajuela, Costa Rica. Febrero, 2017.

Table 1. Average damage degree at eight days after spraying of substances of natural origin for the control of four weed species, by species and herbicide and the probabilities associated with the hypothesis contrasts between species for each herbicide and between herbicides for each species. Estación Experimental Agrícola Fabio Baudrit Moreno (EEAFBM), Alajuela, Costa Rica. Febrero, 2017.

\begin{tabular}{|c|c|c|c|c|c|}
\hline Grado de daño promedio* según: & & & Herbicida & & \\
\hline Especie & $\begin{array}{c}\text { Extracto de } \\
\text { pino** }\end{array}$ & $\begin{array}{l}\text { Extracto de } \\
\text { tomillo**** }\end{array}$ & Glufosinato & D-limoneno & Testigo \\
\hline Amaranthus sp. & 4,75 & 4,25 & 4,00 & 5,00 & 1,00 \\
\hline Bidens pilosa & 2,75 & 3,00 & 4,00 & 5,00 & 1,00 \\
\hline Echinochloa colona & 4,25 & 2,5 & 4,00 & 4,25 & 1,00 \\
\hline Rottboellia cochinchinensis & 4,00 & 4,00 & 4,00 & 4,50 & 1,00 \\
\hline Contrastes evaluados entre especies & & Probal & ad asociada a & ntraste & \\
\hline Hoja ancha vs. Hoja angosta ${ }^{1}$ & 0,07 & 0,07 & 1 & 0,02 & 1 \\
\hline Amaranthus vs. Bidens & 2,3e-09 & $4,7 e-5$ & 1 & 1 & 1 \\
\hline Echinochloa vs. Rottboellia & 0,384 & $2 e-6$ & 1 & 0,38 & 1 \\
\hline Contrastes evaluados entre herbicidas & & Probal & ad asociada a & ntraste & \\
\hline Testigo vs. herbicidas & $1 \mathrm{e}-22$ & $22-17$ & $7 \mathrm{E}-18$ & $2 \mathrm{e}-20$ & No aplica \\
\hline Glufosinato vs. herbicidas naturales & 0,01 & 0,08 & 0,16 & 0,48 & No aplica \\
\hline Limoneno vs. extractos & 0,05 & $5 \mathrm{E}-12$ & 0,0008 & 0,05 & No aplica \\
\hline Extracto de pino vs. extracto de tomillo & 0,85 & 0,38 & $7,2 \mathrm{e}-8$ & 1 & No aplica \\
\hline
\end{tabular}

*1= no existen síntomas aparentes; 2 = presencia de síntomas visuales leves (clorosis leve); $3=$ síntomas visuales moderados (clorosis generalizada); $4=$ síntomas visuales severos (clorosis generalizada, con algo de tejido necrótico); $5=$ síntomas visuales muy severos (similar a 4, pero con frecuente muerte de plantas). **Pinus sp.; ***Thymus vulgaris. $/ 1=$ there are no apparent symptoms; $2=$ presence of mild visual symptoms (mild chlorosis); $3=$ moderate visual symptoms (generalized chlorosis); $4=$ severe visual symptoms (generalized chlorosis, with some necrotic tissue); 5= very severe visual symptoms (similar to 4, but with frequent plant death). **Pinus sp.; ***Thymus vulgaris.

Contrastes entre especies para cada herbicida: a los ocho dda los extractos de pino y tomillo contralaron por igual malezas de hoja ancha o angosta. Los mejores resultados con las cuatro especies ocurrieron con d-limoneno $\mathrm{y}$, aunque presentaron diferencias por tipo de maleza, tuvo un mayor impacto sobre las especies de hoja ancha. El extracto de tomillo controló mejor $R$. cochinchinensis que $E$. colona y ambos extractos controlaron mejor Amaranthus sp. que B. pilosa (Cuadro 1). Similares resultados se observaron a los 15 dda (Cuadro 2). 
Cuadro 2. Grado de daño promedio a los 15 días después de aspersión de sustancias de origen natural para el control de cuatro especies de arvenses, por especie, por herbicida y las probabilidades asociadas a los contrastes de hipótesis entre especies para cada herbicida y entre herbicidas para cada especie. Estación Experimental Agrícola Fabio Baudrit Moreno (EEAFBM), Alajuela, Costa Rica. Febrero, 2017.

Table 2. Average damage degree at 15 days after spraying of substances of natural origin for the control of four weed species, by species and herbicide and the probabilities associated with the hypothesis contrasts between species for each herbicide and between herbicides for each species. Estación Experimental Agrícola Fabio Baudrit Moreno (EEAFBM), Alajuela, Costa Rica. Febrero, 2017.

\begin{tabular}{|c|c|c|c|c|c|}
\hline \multirow{2}{*}{$\begin{array}{l}\text { Grado de daño promedio* según: } \\
\text { Especie }\end{array}$} & \multicolumn{5}{|c|}{ Herbicida } \\
\hline & Extracto de pino* & Extracto de tomillo** & Glufosinato & D-limoneno & Testigo \\
\hline Amaranthus sp. & 5 & 4,25 & 5 & 5 & 1 \\
\hline Bidens pilosa & 2,75 & 3 & 5 & 5 & 1 \\
\hline Echinochloa colona & 4,25 & 2,5 & 4,5 & 4,25 & 1 \\
\hline Rottboellia cochinchinensis & 4,5 & 4 & 5 & 4,5 & 1 \\
\hline Contrastes evaluados entre especies & \multicolumn{5}{|c|}{ Probabilidad asociada al contraste } \\
\hline Hoja ancha vs. Hoja angosta ${ }^{1}$ & 0,03 & 0,08 & 0,25 & 0,0049 & 1 \\
\hline Amaranthus vs. Bidens & $5,00 \mathrm{E}-10$ & 0,0001 & 1 & 1 & 1 \\
\hline Echinochloa vs. Rottboellia & 0,41 & $6,20 \mathrm{E}-06$ & 0,1 & 0,41 & 1 \\
\hline Contrastes evaluados entre herbicidas & \multicolumn{5}{|c|}{ Probabilidad asociada al contraste } \\
\hline Testigo vs. herbicidas & $3,00 \mathrm{E}-23$ & $5,00 \mathrm{E}-18$ & $1,00 \mathrm{E}-17$ & $2,00 \mathrm{E}-21$ & No aplica \\
\hline Glufosinato vs. herbicidas naturales & 0,32 & $3,40 \mathrm{E}-07$ & 0,0013 & 0,0091 & No aplica \\
\hline Limoneno vs. extractos & 0,16 & $3,00 \mathrm{E}-11$ & 0,0015 & 0,34 & No aplica \\
\hline Extracto de pino vs. extracto de tomillo & 0,02 & 0,41 & $2,90 \mathrm{E}-07$ & 0,1 & No aplica \\
\hline
\end{tabular}

*Pinus sp.; **Thymus vulgaris.

Contrastes entre herbicidas para cada especie: a los ocho dda, para todas las especies, los herbicidas en promedio produjeron un grado de daño que los hizo significativamente diferentes al testigo. Los herbicidas naturales ocasionaron el mismo grado de daño que el glufosinato, con excepción del limoneno, cuyo daño fue mayor a todos. Los grados promedio de daño de los extractos de pino y de tomillo fueron inferiores para E. colona y B. pilosa (Cuadro 1). A los 15 dda se mantuvo el efecto significativo de los herbicidas cuando se comparan con el testigo. Durante esta evaluación, el limoneno mostró efecto similar al glufosinato sobre las arvenses evaluadas. El limoneno mostró mejores resultados que los extractos en B. pilosa y en E. colona. En Amaranthus sp. y en E. colona fue mejor el extracto de pino que el de tomillo (Cuadro 2).

\section{Número de plantas muertas}

A los ocho dda la interacción tipo de herbicida* tipo de arvense no fue significativa ( $p>0,9012)$. Tampoco se encontró significancia en el número de plantas muertas si se comparan los diferentes tipos de arvenses ( $>00,9984)$ y los diferentes tipos de producto $(\mathrm{p}>0,9999)$. Esto pudo ocurrir debido a que a los ocho dda las plantas todavía tenían tejido verde, por lo que aún no se contabilizaron como muertas. En contraste con esto, a los 15 dda todas las razones de ventaja de plantas muertas versus plantas vivas, en las que se compara el testigo contra uno de los herbicidas naturales, resultaron significativas y sustancialmente menores que 1 , con tres excepciones que indicaron que el extracto de pino y el extracto de tomillo no tienen efecto sobre B. pilosa, mientras que el extracto de tomillo 
no tuvo efecto sobre E. colona. En todos los casos, con las excepciones antes mencionadas, se murieron menos plantas en el testigo que en los potes tratados con los herbicidas naturales. En los demás, la proporción de plantas muertas fue significativamente mayor cuando se aplicó uno de los herbicidas (Cuadro 3).

Cuadro 3. Sustancias de origen natural para el control de cuatro especies de arvenses, razones de ventaja de planta muerta vs. viva (OR), a los 15 dda. Estación Experimental Agrícola Fabio Baudrit Moreno (EEAFBM), Alajuela, Costa Rica. Febrero 2017.

Table 3. Substances of natural origin for the control of four weed species, advantage reasons for dead plant vs alive (OR), at 15 das. Estación Experimental Agrícola Fabio Baudrit Moreno (EEAFBM), Alajuela, Costa Rica. February 2017.

\begin{tabular}{|c|c|c|c|c|c|c|c|c|}
\hline \multirow{2}{*}{$\begin{array}{l}\text { Número de plantas muer- } \\
\text { tas promedio* según: } \\
\text { Herbicidas }\end{array}$} & \multicolumn{8}{|c|}{ Especie } \\
\hline & \multicolumn{2}{|c|}{ Amaranthus sp. } & \multicolumn{2}{|c|}{ B. pilosa } & \multicolumn{2}{|c|}{ E. colona } & \multicolumn{2}{|c|}{ R. cochinchinensis } \\
\hline Extracto de pino* & \multicolumn{2}{|c|}{5} & \multicolumn{2}{|c|}{0,25} & \multicolumn{2}{|c|}{2,25} & \multicolumn{2}{|c|}{3,25} \\
\hline Extracto de tomillo** & \multicolumn{2}{|c|}{4,25} & \multicolumn{2}{|c|}{0} & \multicolumn{2}{|c|}{0,25} & \multicolumn{2}{|c|}{1} \\
\hline Glufosinato & \multicolumn{2}{|c|}{6} & \multicolumn{2}{|c|}{6} & \multicolumn{2}{|c|}{0,5} & \multicolumn{2}{|c|}{2,25} \\
\hline D-limoneno & \multicolumn{2}{|c|}{5,25} & \multicolumn{2}{|l|}{5,75} & \multicolumn{2}{|c|}{3} & \multicolumn{2}{|c|}{4,5} \\
\hline Testigo & \multicolumn{2}{|c|}{0} & \multicolumn{2}{|c|}{0} & \multicolumn{2}{|c|}{0} & \multicolumn{2}{|c|}{0} \\
\hline Ventaja de muerta vs. viva & OR & $\mathbf{P}^{1}$ & OR & $\mathbf{P}^{1}$ & OR & $\mathbf{P}^{1}$ & OR & $\mathbf{P}^{1}$ \\
\hline E. tomillo vs. E. pino & 0,49 & 0,3005 & $1,05 \mathrm{E}-07$ & 0,24 & 0,07 & $0,0026^{*}$ & 0,17 & $0,0056^{*}$ \\
\hline Glufosinato vs. E.pino & 43726149 & $0,0151 *$ & $5,03 \mathrm{E}+09$ & $<, 0001^{*}$ & $3,644 \mathrm{E}+09$ & $<, 0001 *$ & 184995245 & $<, 0001 *$ \\
\hline Glufosinato vs. E.tomillo & 90024424 & $0,0010^{*}$ & $4,78 \mathrm{E}+16$ & $<, 0001^{*}$ & $5,03 \mathrm{E}+09$ & $<, 0001 *$ & $1,09 \mathrm{E}+09$ & $<, 0001 *$ \\
\hline Limoneno vs. E. pino & 1,4 & 0,6821 & 529 & $<, 0001^{*}$ & 1,67 & 0,38 & 7,62 & $0,0069 *$ \\
\hline Limoneno vs. E. tomillo & 2,88 & 0,15 & $5,03 \mathrm{E}+09$ & $<, 0001 *$ & 23 & $0,0001 *$ & 45 & $<, 0001 *$ \\
\hline Limoneno vs. Glufosinato & $3,20 \mathrm{E}-08$ & $0,0368^{*}$ & $1,05 \mathrm{E}-07$ & 0,24 & 4,57E-09 & $<, 0001 *$ & $4,12 \mathrm{E}-08$ & 0,07 \\
\hline Testigo vs. E. pino & $9,15 \mathrm{E}-10$ & $<, 0001^{*}$ & $1,05 \mathrm{E}-07$ & 0,24 & $7,62 \mathrm{E}-09$ & $0,0001 *$ & 3,87E-09 & $<, 0001 *$ \\
\hline Testigo vs. E. tomillo & $1,88 \mathrm{E}-09$ & $<, 0001 *$ & 1 & . & $1,05 \mathrm{E}-07$ & 0,24 & $2,29 \mathrm{E}-08$ & $0,0151^{*}$ \\
\hline Testigo vs. Glufosinato & $2,09 \mathrm{E}-17$ & $<, 0001 *$ & $2,09 \mathrm{E}-17$ & $<, 0001^{*}$ & $2,09 \mathrm{E}-17$ & $<, 0001 *$ & $2,09 \mathrm{E}-17$ & $<, 0001^{*}$ \\
\hline Testigo vs. Limoneno & $6,53 \mathrm{E}-10$ & $<, 0001^{*}$ & $1,99 \mathrm{E}-10$ & $<, 0001 *$ & 4,57E-09 & $<, 0001 *$ & $5,08 \mathrm{E}-10$ & $<, 0001 *$ \\
\hline
\end{tabular}

*Pinus sp.; **Thymus vulgaris.

${ }^{1}$ Amaranthus sp. y B. pilosa son especies de hoja ancha y E. colona y R. cochinchinensis son especies de hoja angosta / ${ }^{1}$ Amaranthus sp. and B. pilosa are broadleaf species, and E. colona and R. cochinchinensis are the narrow leaf species.

En todas las especies el glufosinato tuvo mayor efecto fitotóxico en comparación con el extracto de pino y el extracto de tomillo, y lo mismo ocurrió con el limoneno para Amaranthus sp. y E. colona. El limoneno tuvo el mismo efecto que el glufosinato para B. pilosa y R. cochinchinensis, las razones de ventaja no resultaron significativamente diferentes de 1 , sin embargo, el orden de magnitud de las razones de ventaja en la muestra $(1,052 \mathrm{e}-7$ y 4,1165e-8) hizo que la conclusión no fuera contundente. El limoneno mostró ventaja significativa sobre el extracto de pino y sobre el extracto de tomillo en B. pilosa y en R. cochinchinensis y sobre el extracto de tomillo en E. colona. En Amaranthus sp., a pesar de que las razones de ventaja fueron superiores a 1 en la muestra, este resultado no fue significativo. Al comparar el extracto de tomillo con el extracto de pino se encontraron desventajas significativas del tomillo contra el pino en E. colona y en R. cochinchinensis (Cuadro 2). 


\section{Discusión}

La eficacia encontrada de los herbicidas naturales en este trabajo, concuerda con lo que reportaron otros autores. En el caso de limoneno se reportó la inhibición del crecimiento de la arvense Amaranthus viridis, dado que la acción de este compuesto podría afectar la actividad mitótica de las arvenses (Vaid et al., 2011). La reducida eficacia del extracto de pino sobre B. pilosa, pudo deberse a que este extracto se utiliza, en la mayoría de los casos, con efecto inhibitorio sobre la germinación de las arvenses (Jiménez-Ferrer et al., 2006). En cuanto al extracto de tomillo, también se reportó su fitotoxicidad sobre la especie Amaranthus retroflexus y su efecto sobre esta especie fue más eficaz que la acción del 2,4-D (Dayan et al., 2015). Si bien la literatura reporta que el extracto de tomillo contiene timol y p-cymene, ambos son compuestos fitotóxicos (Dayan et al., 2015) y su baja actividad encontrada en este trabajo, podría deberse a que se requiere una mayor dosis para ejercer un control eficaz.

El rápido desarrollo de síntomas al aplicar los herbicidas naturales en comparación con el glufosinato, estuvo asociado a la presencia de ácidos grasos en este tipo de sustancias naturales, los cuales se encuentran involucrados en disolución de la cera cuticular de las hojas, lo que desencadena una rápida desestabilización de la membrana plasmática (Amri et al., 2013). En otros estudios también se reportó daño en las arvenses al comparar un herbicida sintético con respecto a una sustancia natural; el butaclor y el extracto de A. conyzoides no tuvieron diferencias significativas entre sí (Xuan et al., 2004). Las sustancias naturales utilizadas poseen actividad herbicida y en algunos casos fueron similar a un herbicida sintético.

\section{Conclusiones}

El d-limoneno fue el herbicida más eficaz en comparación con el extracto de pino y el extracto de tomillo sobre las arveneses, Bidens pilosa, Amaranthus sp., Echinochloa colona y Rottboellia cochinchinensis.

Al comparar la eficacia del glufosinato con los herbicidas naturales, se encontró que el d-limoneno produjo mejores resultados al inicio de la evaluación, sin embargo, en la última evaluación ambos herbicidas produjeron efectos similares.

\section{Referencias}

Agro Research International. (2020). Thyme Guard@: Bactericida, fungicida e insecticida para uso en todos los cultivos alimenticios y no alimenticios. Agro Research International. https://www.agroresearchinternational.com/es/thyme-guard

Amri, I., Hamrouni, L., Hanana, M., \& Jamoussi, B. (2013). Reviews on phytotoxic effects of essential oils and their individual components: news approach for weeds management. International Journal of Applied Biology and Pharmaceutical Technology, 4, 96-114.

Avenger. (s.f). Welcome to Avenger® organics! Avenger Products, LLC. http://www.avengerorganics.com/

Bailey, K. L. (2014). The bioherbicide approach to weed control using plant pathogens. In D. P. Abrol (Ed.), Integrated Pest Management (pp. 245-266). Academic Press. https://doi.org/10.1016/B978-0-12-398529-3.00014-2

Bayer AG. (2020). Basta@ 14 SL. Bayer CropScience. https://www.sag.gob.cl/sites/default/files/basta_14_sl_01-04-2020.pdf

Bhowmik, P. C., \& Inderjit, D. (2003). Challenges and opportunities in implementing allelopathy for natural weed management. Crop protection, 22(4), 661-671. https://doi.org/10.1016/S0261-2194(02)00242-9 
Blanco, Y. (2006). La utilización de la alelopatía y sus efectos en diferentes cultivos agrícolas. Cultivos Tropicales, $27(3), 5-16$. https://www.redalyc.org/pdf/1932/193215825001.pdf

Castillo, M. D. P., \& Gómez, R. (2016). Efecto de la esterilización del suelo con vapor de agua sobre semillas de malezas. Agronomía Mesoamericana, 27(2), 409-413. https://doi.org/10.15517/am.v27i2.21286

Dayan, F. E., Cantrell, C. L., \& Duke, S. O. (2009). Natural products in crop protection. Bioorganic \& Medicinal Chemistry, 17(12), 4022-4034. https://doi.org/10.1016/j.bmc.2009.01.046

Dayan, F. E., \& Duke, S. O. (2014). Natural compounds as next-generation herbicides. Plant Physiology, 166(3), $1090-1105$. https://doi.org/10.1104/pp.114.239061

Dayan, F. E., Owens, D. K., Watson, S. B., Asolkar, R. N., \& Boddy, L. G. (2015). Sarmentine, a natural herbicide from Piper species with multiple herbicide mechanisms of action. Frontiers in Plant Science, 6, 222, 1-11. https://doi. org/10.3389/fpls.2015.00222

Dotolo, V. (1983). Pesticides containing D-limonene. (U.S. Patent No 4,379,168). PubChem. https://pubchem.ncbi.nlm.nih.gov/ patent/US-4379168-A

Duke, S. O., \& Lydon, J. (1987). Herbicides from natural compounds. Weed technology, 1(2), 122-128. https://doi.org/10.1017/ S0890037X00029304

Duke, S. O., Romagni, J. G., \& Dayan, F. E. (2000). Natural products as sources for new mechanisms of herbicidal action. Crop Protection, 19(8-10), 583-589. https://doi.org/10.1016/S0261-2194(00)00076-4

Enlasa. (s.f.) Pegador RP 10 SL. Grupo Enlasa productos. https://grupoenlasa.com/pegador-rp-10-sl/

Hernández, E. A., \& Álvarez, R. (2008). Uso de los extractos acuosos del pino macho (Pinus caribaea Morelet) en el control de las malezas en cafetales bajo sombra (Accession No. 3194). Fitosanidad, 12(3), 184. http://www.sidalc.net/cgi-bin/ wxis.exe/?IsisScript=pubs. $x$ is \&method=post $\&$ formato $=2 \&$ cantidad $=1 \&$ expresion $=m f n=002424$

Jabran, K., Mahajan, G., Sardana, V., \& Chauhan, B. S. (2015). Allelopathy for weed control in agricultural systems. Crop Protection, 72, 57-65. https://doi.org/10.1016/j.cropro.2015.03.004

Jiménez-Ferrer, L. J., Valdés, D., \& Álvarez, R. (2006). Efecto alelopático de Pinus caribaea en la germinación de arvenses en casas de cultivo protegido. Centro Agrícola, 33(4), 79. http://cagricola.uclv.edu.cu/descargas/pdf/V33-Numero_4/ cag144061519.pdf

Masschelein, L. (2004). Les solventes. Centro Nacional de Conservación y Restauración. http://www.ibermuseos.org/wpcontent/uploads/2020/05/los-solventes-chi.pdf

Messerschmidt, O., \& Jankauskas, J. (2012). U.S. Patent No. 8,153,561. U.S. Patent and Trademark Office.

Sandral, G. A., Dear, B. S., Pratley, J. E., \& Cullis, B. R. (1997). Herbicide dose rate response curves in subterranean clover determined by a bioassay. Australian Journal of Experimental Agriculture, 37(1), 67-74. https://doi.org/10.1071/ EA96067

Shrestha, A., Moretti, M., \& Mourad, N. (2012). Evaluation of thermal implements and organic herbicides for weed control in a nonbearing almond (Prunus dulcis) orchard. Weed Technology, 26(1), 110-116. https://doi.org/10.1614/WT-D-1100083.1

Skat orgánico. (s.f.). Riso-fort. Skat limitada. http://www.skatlimitada.cl/ 
Tworkoski, T. (2002). Herbicide effects of essential oils. Weed Science, 50(4), 425-431. https://doi.org/10.1614/00431745(2002)050[0425:HEOEOfont>2.0.CO;2

Vaid, S., Batish, D. R., Singh, H. P., \& Kohli, R. K. (2011). Phytotoxicity of limonene against Amaranthus viridis L. Bioscan, $6(1), 163-165$.

Valverde, B. E., \& Heap, I. M. (2009, noviembre). El estado actual de la resistencia a herbicidas en el mundo (Serie Actas). Instituto de Investigaciones Agropecuarias. Recuperado el 13 marzo, 2021, de https://biblioteca.inia.cl/bitstream/ handle/123456789/8572/NR36351.pdf?sequence=1\&isAllowed=y

Xuan, T. D., Shinkichi, T., Hong, N.H., Khanh, T.D., \& Min,C. I. (2004). Assessment of phytotoxic action of Ageratum conyzoides L. (billy goat weed) on weeds. Crop Protection, 23(10), 915-922. https://doi.org/10.1016/j.cropro.2004.02.005. 